\title{
Working Together towards the Sub-mSv CT Exam
}

\section{Denison K}

Radiation dose from CT - It's a concern that many share-clinicians, manufacturers, patients, and government agencies. While recent events have highlighted the importance of reducing medical radiation dose in the general population and news media, it is an issue that GE Healthcare has addressed for over 30 years.

At GE Healthcare, patient safety is our primary concern.

Consider the sheer number of CT exams performed each year. According to the latest CT census data from $\mathrm{IMV}^{1}$, an estimated 68.7 million $\mathrm{CT}$ procedure were performed in 7,640 hospital and non-hospital sites in 2007. This represents a growth of approximately $8 \%$ since 2003 . During this same time, multi-detector CT became the standard of care in many clinical care areas, causing some to raise concerns over the potential for inappropriate radiation from CT imaging.

There is a certain irony with the use of CT imaging. Medical radiation in imaging technologies has enabled major medical advancements in the accurate detection of disease, the delivery of treatment, and the evaluation of treatment efficacy. They allow physicians to more easily visualize and characterize disease without the need for invasive procedures.

In fact, a recent study by Columbia University Professor of Business Frank
Lichtenberg and based on data from the National Cancer Institute and Thomson Medstat, found that medical imaging innovation accounted for $40 \%$ of the decline in cancer deaths in the US over the last decade. And, CT imaging has been called one of the most significant advances in the history of healthcare.

Yet, along with these powerful contributions to the quality and advancement of healthcare delivery, the issue of medical radiation also requires that physicians, healthcare facilities, and manufacturers work together to develop and evolve effective processes and systems to promote its appropriate use.

Medical radiation in imaging technologies has enabled major medical advancements in the accurate detection of disease, the delivery of treatment, and the evaluation of treatment efficacy.

At GE Healthcare, we believe that this responsibility goes beyond the development and evolution of dose-reduction technologies. We support a comprehensive approach that takes into account the environment in which such equipment is used and those who are responsible for its implementation. We believe that a collaborative, system-wide approach to creating a dose-conscious healthcare system will help promote positive change-without limiting the progress of healthcare and its stewards to serve their patients well. 


\section{Starting with research}

Promoting the best use of CT imaging is an important first step in lowering medical radiation dose. We believe, as a manufacturer, it is not enough to just build a better machine and hope it is used properly - we also have an invested stake in promoting the optimal use of the technology we make to help support the best patient care.

To that end, GE Healthcare is spending millions of dollars to fund research to identify and help define the standards, practices, and metrics required for any healthcare provider to implement a safe, effective, and efficient CT imaging program. To conduct this research in an independent and unbiased manner, GE has provided a grant to a third party, non-profit research organization focused on improving patient safety. Through collaboration with multiple physician researchers across multiple care settings - from small, rural hospitals to the largest, most complex academic medical centers - the organization will collect and analyze procedural data, practices, protocols, processes, and financial and outcomes data for the full imaging cycle in order to develop a consensus on the "is state" and the "best achievable state" of CT care.

The objectives of this research are to:

- Provide a forum for endorsement;

- Document the current "is state" of CT imaging across multiple care settings;

- Define the "best achievable state" across multiple care settings;

- Identify opportunities to help close the gap between the "is" and "best achievable" states;
- Address the opportunities for highperformance improvement around the full loop from patient assessment for imaging ordering, image execution, and reporting in the new valuebased purchasing environment.

Driving this research is the idea that highperformance imaging programs ensure that the right procedure is done in the right way at the right time with the right dose, and the correct interpretation is accurately and quickly communicated to the patient. The results of this project will be published in the literature so that all stakeholders can benefit from them.

GE Healthcare believes that highperformance imaging programs occur only when a system-wide approach is taken and only when the program correctly addresses three key dimensions:

Leadership - the policies, processes, metrics, performance systems, culture, and change management that are characteristics of high performers;

Practices - the use of the best practices including appropriateness criteria, triage, protocol development, and scanning procedures; and

Technologies - the use of the right CT devices, dose-lowering features and dose reporting/ tracking/ monitoring software.

A key output of the program will be assets to help healthcare providers assess their current leadership, practices, and technologies and assess gaps in the four "A's": awareness, accountability, ability, and action. Are we aware of performance gaps, best practices, 
and the benefits and risks of current and new technologies? Are there leaders or clinicians accountable to close gaps and adopt best practices? Do our leaders, clinicians, and staff members have the ability and knowhow to close the gaps and ensure patient safety? Are we consistently taking action to close our gaps?

\section{Solutions to close the gap}

At GE Healthcare, our intent is to help our customers' buildhigh performance imaging programs using this system-wide approach. Our focus is not only on the CT scanning technology we build, but on additional products and services that will help our customers build better imaging programs.

Today, GE Healthcare is working on DoseWatch, a new IT application that is expected to be commercially available this year. The goal is to allow clinicians to track, report, and monitor dose from multiple manufacturers and multiple imaging devices, including CT, interventional, mammography, and R\&F. Our customers will have one source of radiation dose information that can be used to optimize their performance, monitor sensitive patients, and help prevent errors.

Also look for Dose Check coming to GE Healthcare CT scanners this year. In response to an FDA request that stemmed from the Agency's investigation into recent incidents of overexposure, the Medical Imaging and Technology Alliance (MITA) a trade group comprised of the five major manufacturers of CT systems - developed Dose Check, an industry standard to provide healthcare providers with tools to set limits on the amount of radiation that will be delivered during CT scans.

The objective of Dose Check is to ensure there are notifications and alerts provided to the operator before a CT scan is prescribed above pre-determined radiation exposure levels.

\section{How low can dose go?}

In addition to adopting the new Dose Check standard, GE will continue its 30-year commitment to developing technologies that can lower dose without impacting image quality. Over 900 installed GE CT scanners today are equipped with ASiR (Adaptive Statistical Iterative Reconstruction), a proven technique for lowering dose while maintaining image quality. ASiR has been used in over 7 million exams to date.

Our next generation dose reduction technique, Veo shows great promise in providing exceptional image quality at even lower doses. Available today in markets outside the US, Veo is helping clinical users move toward our shared goal of routine, sub$\mathrm{mSv}$ CT exams. Early adopters are seeing great results with Veo and we look forward to continuing to share their clinical cases with you in future issues of CT Clarity.

And there is still more to come. At GE Healthcare we recently completed our annual strategic planning process. Featured prominently in this year's plan are still more dose-lowering features for our CT devices and new services to help build a high performance CT imaging program. We look forward to telling you more about these exciting developments in future CT Clarity articles. 
Lowering dose isn't just about building a better device. It's about working together, to ensure that every patient receives the right exam, at the right time, and at the right dose.

\section{Dose Check}

Dose Check is a NEMA XR-25 standard defined in conjunction with MITA and other stakeholders that notifies and alerts the operating personnel, generally technologists, when the estimated dose index is above the value defined and set by the operating group, practice, or institution. It is part of a department's Quality Assurance process providing tools for auditing and tracking scans where Alert or Notification values have been exceeded.

Notification Value (NV) is the dose index that is above the institution's established range for the protocol. $\mathrm{NV}$ is intended to be set at a level that would be considered above "routine" or "normally expected" dose, but not at such a high level as to pose a significant risk to the patient.Depending on the patient size or imaging need it may be appropriate to scan at a value above the notification value in order to achieve the diagnostic intent of the exam. Scanning is possible with no further authorization if the $\mathrm{NV}$ level is exceeded. GE encourages sites to establish appropriate notification values for all scanning. The AAPM has published a list of reference NV's on its website at www.aapm.org. Alert Value (AV) is a value established by the institution which will trigger an alert when the prescribed imaging series would cause the dose accumulated over the course of the exam to exceed this value. It is checked at exam level according to system presets and requires a user with authorization to confirm a scan exceeding this value. Manufacturers have pre-populated the AV at 1,000 mGy in accordance with FDA suggestion. 